\title{
Neck Circumference Marker for Metabolic Syndrome in Menopause Transition
}

\author{
N. Hephzibah Kirubamani* \\ Obstetrics and Gynaecology, Saveetha University, Chennai - 600077, Tamil Nadu, \\ India; hepsi1002@yahoo.co.in
}

\begin{abstract}
Introduction: According to few studies carried out among peri-menopause women there is increase in weight and in hip circumference. These changes can increase the risk of Metabolic Syndrome. Upper body fat distribution can be easily be identified by Neck Circumference (NC). Objective: To find out whether NC can be used to predict Metabolic Syndrome in peri-menopause transition. Methods: A cross sectional, observational prospective study was conducted at Saveetha Medical College, Obstetrics and Gynaecology Outpatient Department. A total of 580 women were recruited randomly belonging to the age group between 35 years to 60 years and divided into pre, peri- and post-menopause women. NC was correlated with Metabolic Syndrome based on the National Cholesterol Education Program, Adult Treatment Panel III. Results: Out of the 580 women, 178 (31\%) belonged to pre-menopause, 198 (34\%) to peri-menopause and 204 (35\%) to post-menopause women. When the mean NC was $34.04 \pm 3.69 \mathrm{~cm}$ Metabolic Syndrome was absent. Metabolic Syndrome was present in 313 (54\%) of women and the mean NC was $36.64 \pm 2.56 \mathrm{~cm}$. Out of these, 139 (24\%) women belonged to peri-menopause age group. When NC was abnormal it correlated well with all parameters of Metabolic Syndrome namely BMI, Waist Circumference, WHR, Fasting Blood Glucose, Total Cholesterol, Triglycerides, HDL and LDL. Cut-off of for NC was $36.64 \mathrm{~cm}$ which had a Sensitivity of $86.9 \%$ and a specificity of $74.15 \%$ with a Positive Predictive Value of $79.7 \%$ and a Negative Predictive Value of $85.7 \%$ Conclusion: Neck Circumference is a simple and easy screening test and marker for metabolic syndrome in peri-menopause women.
\end{abstract}

Keywords: Menopause Transition, Metabolic Syndrome, Neck Circumference, Upper Body Fat, Screening Test

\section{Introduction}

Prevalence of obesity and Metabolic Syndrome is known to increase during the midlife of women. Gaining of weight around the abdomen, rather than around the hips and thighs occurs in the peri-menopause age group and this may be due to hormonal changes. Additionally, it may be related to aging, lifestyle and genetic factors. Metabolic Syndrome occurs in menopause due to change in hormonal milieu. To compensate for the decrease in oestrogen, in peri-menopause period, women are likely to gain weight around the abdomen and about $80 \%$ of women gain weight. This central obesity causes increased levels of pro-inflammatory cytokines, increased visceral fat and decreased adiponectin, increased free fatty acids which subsequently increases insulin resistance resulting in Hyperglycaemia, Hypertension and Dyslipidaemia.

A few studies have found increase in weight and in hip circumference among peri-menopause women and abdominal fat may increase in peri-menopausal years $\stackrel{1}{\text {. }}$ Fat deposition in the abdomen in this age group causes insulin resistance and subsequent Diabetes Mellitus and heart disease and peri-menopause age group are predisposed to comorbid conditions ${ }^{2}$. Fat in the unusual site, namely the upper body, can act as a reserve and releases immediate energy ${ }^{3}$. Upper body mass releases 
free fatty acids much more than visceral fat, which can cause insulin resistance ${ }^{4}$.

Increased release of free fatty acids causes oxidative stress, which in turn leads to metabolic and cardiovascular risk ${ }^{5}$. Upper body subcutaneous fat can be estimated by Neck Circumference and can be used for screening for overweight and obesity.

Body Mass Index (BMI), Waist Circumference (WC), Waist-to-Hip Ratio (WHR) and Waist-to-Height ratio (WHR) are simple Anthropometric Indices that are widely used to detect central obesity and predict Metabolic Syndrome. Nowadays, Neck Circumference (NC) is considered as a simple and easy measurement tool to predict central obesity and Metabolic Syndrome. To determine body fat distribution, Neck Circumference is an alternative and better approach ${ }^{6}$. NC is better than waist circumference in predicting metabolic factors. NC, which reflects upper body obesity, has strong association with Glucose Intolerance, Hyper-insulinaemia and Hyper-triglyceridemia.

\section{Objective}

This study was done to determine the association of Neck Circumference (NC) and Metabolic Syndrome during Menopause Transition.

\section{Materials and Methods}

Cross sectional observational prospective study was conducted at Saveetha Medical College, Obstetrics and Gynaecology Outpatient Department. 580 women who visited the Gynaec Department of the hospital, between the age group from 35 years to 60 years were recruited by a simple random technique. Women with medical disorders and women with goitre, lymph node enlargement and neck swelling, were excluded. The women were divided into three groups: Pre-menopausal 35-40 years, perimenopausal 41-50 years and post-menopausal 51-60 years.

\subsection{Measurements}

After eliciting their gynaec history and after physical examination, informed consent was obtained from the subjects. All cardio-metabolic risk factors were assessed. All measurements were recorded according to the guidelines laid down by the WHO. NC was measured with a flexible tape in a standing position with the head in an erect position with the eyes facing forward. The measurements were made below the thyroid cartilage in front (the upper margin of the laryngeal prominence - Adam's apple) and at the level of mid cervical spine at the back. Weight was measured using a digital scale and height was determined using a portable stadiometer. Waist circumference was measured using flexible tape at midpoint between the lowest rib and iliac crest after gentle expiration. Hip circumference was measured around the greatest circumference of the buttock using flexible tape with subject standing with light clothes. BMI (weight in kilograms divided by height in meters squared $\left[\mathrm{kg} / \mathrm{m}^{2}\right]$ ) was calculated. Waist Hip Ratio (WHR) - (WC divided by $\mathrm{HC}$ ) values were calculated. After a 5-minute rest, blood pressure was measured in a sitting position. After overnight fasting, blood samples were collected and sent to the laboratory attached to the hospital for the determination of Fasting Blood Sugar, Total Cholesterol (TC) and Triglyceride (TG) concentrations, High-density Lipoprotein Cholesterol (HDL-C) and Low-density Lipoprotein Cholesterol (LDL-C).

The definition of MS we adopted in this study was based on the criteria for the metabolic syndrome laid down by the National Cholesterol Education Program, Adult Treatment Panel III. They consisted of three or more of the following criteria:

- Elevated waist circumference: Greater than 35 inches $(88 \mathrm{~cm})$.

- Elevated triglycerides: Equal to or greater than 150 $\mathrm{mg} / \mathrm{dL}$.

- HDL: Less than $50 \mathrm{mg} / \mathrm{dL}$.

- Elevated blood pressure Equal to or greater than $130 / 85 \mathrm{~mm} \mathrm{Hg}$.

- Elevated fasting glucose: Equal to or greater than 100 $\mathrm{mg} / \mathrm{dL}$.

\subsection{Statistical Significance}

Statistical analysis was carried out using Chi Square and Karl Pearson Correlation Coefficients. The significance analysis was carried out using Chi Square Test for the Identified distribution of Population with different age group for metabolic syndrome. Pearson's Correlation Coefficients were used to analyse the interrelationships between anthropometric indexes 
and age, weight and waist circumference, triglycerides, HDL, blood pressure and fasting glucose. Multiple Regression Analysis was adopted for analysing the relative contributions of the variability of plasma lipids, glucose and uric acid to changes in NC and other anthropometric and demographic changes. The pooled form of statistics tested equality of variance and correction for unequal variances was performed where appropriate.

The independent variables used in the model were demographic, anthropometric and biochemical data that were recorded initially and their relative changes. A stepwise selection method was used to delineate a set of contributory variables to ascertain the variability of risk factors. Chi square test was conducted with a Confident Interval of $95 \%$. Only after this, a check was made and necessary deletions accomplished, that another variable could be added to the model. The relative contribution of an independent (predictor) variable to the relative change of a risk factor was measured by Partial Squared Correlation.

\section{Results}

The study sample consisted of 580 women between the ages of 35 years to 60 years. 178 (31\%) women from the 35-40 years age group were in the pre-menopause stage, 198 (34\%) women from the 41-50 years age group belonged to the peri-menopause group and 204 (35\%) women from the 51-60 years came from the post-menopause set (Figure 1).

In this study, Metabolic Syndrome was absent when the mean Neck Circumference (NC) was 34.04 $\pm 3.69 \mathrm{~cm}$. The Mean Neck Circumference (NC) was $36.64 \pm 2.705 \mathrm{~cm}$. among women who had Metabolic Syndrome, with $\mathrm{p}$ a value of $<0.001$ and the correlation is statistically significant. Out of the 580 women, 313 (54\%) had Metabolic Syndrome and in 267 (46\%) women, Metabolic Syndrome was absent (Table 1). Metabolic Syndrome was seen in 52 (9\%) of premenopause women, 139 (24\%) of peri-menopause women and $122(21 \%)$ of post-menopause women (Figure 2). In our study when NC is abnormal, it correlated well with all anthropometric measurements namely, BMI, WC, HC and WHR. There was good correlation with Systolic, Diastolic, Blood Pressure, Fasting Blood Glucose, HDL and Triglycerides, indicating presence of Metabolic Syndrome in that group (Table 2). In this study, mean Cut off value $36.64 \mathrm{~cm}$ of NC identifies with Metabolic Syndrome with a sensitivity of $86.9 \%$ and a specificity of $74.15 \%$ with Positive predictive value of $79.7 \%$ and Negative predictive value $85.7 \%$ (Table 3 ).

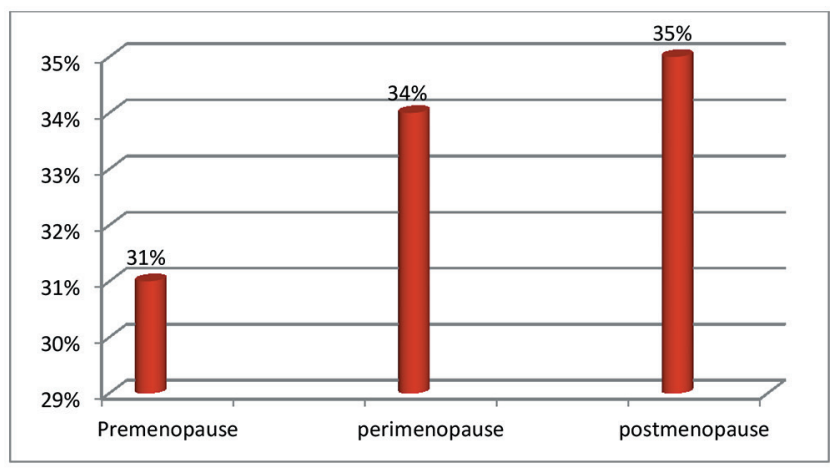

Figure 1. Distribution of the cases shows 178 (31\%) women from 35-40 years age group belong to pre-menopause, 198 (34\%) women from 41-50 years age group belong to perimenopause and 204 (35\%) women from 51-60 years - postmenopause age group.

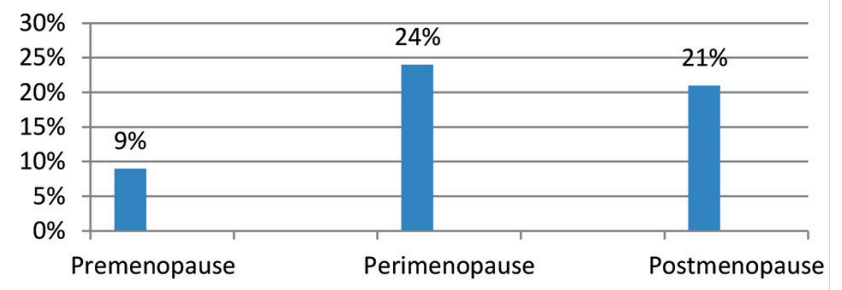

Figure 2. Distribution of Metabolic Syndrome in various age groups. Metabolic syndrome was seen higher among peri-menopause women Metabolic Syndrome absent when mean Neck Circumference is NC $34.04 \mathrm{~cm}(+/-3.69)$. When Mean Neck Circumference (NC) is $36.64 \mathrm{~cm}(+/-2.705)$ metabolic syndrome was present.

Table 1. Mean Neck Circumference and Association of Metabolic Syndrome

\begin{tabular}{|c|c|c|c|c|}
\hline $\begin{array}{c}\text { Metabolic } \\
\text { Syndrome }\end{array}$ & $\begin{array}{c}\text { Number } \\
\text { of cases }\end{array}$ & Mean NC & t-value & p-value \\
\hline Absent & 267 & $34.04+/-3.9$ & 7.534 & $<0.001$ \\
\hline Present & 313 & $36.4+/-2.6$ & & \\
\hline
\end{tabular}


Table 2. Neck Circumference its association with Metabolic Syndrome

\begin{tabular}{|l|c|c|}
\hline $\begin{array}{c}\text { Factors for Metabolic } \\
\text { syndrome }\end{array}$ & $\begin{array}{c}\text { Karl Pearson } \\
\text { correlation } \\
\text { co-efficient r value }\end{array}$ & P value \\
\hline Age & 0.031 & 0.541 \\
\hline BMI & .483 & $<0.001$ \\
\hline Waist Circumference & .448 & $<0.001$ \\
\hline WHR & .521 & $<0.001$ \\
\hline SBP & .232 & $<0.001$ \\
\hline DBP & .104 & $<0.001$ \\
\hline Total cholesterol & .261 & $<0.001$ \\
\hline HDL & .172 & $<0.001$ \\
\hline LDL & .342 & $<0.001$ \\
\hline Fasting Glucose & .162 & $<0.001$ \\
\hline
\end{tabular}

When NC is abnormal it is correlated well with all anthropometric and all factors of Metabolic Syndrome

Table 3. NC Sensitivity, Specificity, Positive Predictive Value and Negative Predictive Value in Predicting Metabolic Syndrome

\begin{tabular}{|c|c|}
\hline Mean NC Cut off $36.64 \mathrm{~cm}$ & Percentage \\
\hline Sensitivity & $86.9 \%$ \\
\hline Specificity & $74.15 \%$ \\
\hline PPV & $79.7 \%$ \\
\hline NPV & $85.7 \%$ \\
\hline
\end{tabular}

Mean cut off value $36.64 \mathrm{~cm}$ of NC, Metabolic Syndrome identified with the sensitivity of $86.9 \%$ and a specificity of $74.15 \%$ with Positive predictive value of $79.7 \%$ and Negative predictive value.

\section{Discussion}

There are many methods to assess overweight and obesity (abdominal obesity). Out of them, few methods use parameters like weight, height, abdominal and waist circumference from which it is possible to calculate BMI and waist hip ratio. However, in a busy outpatient department, it is rather difficult to measure all these parameters. It is also not feasible to take these measurements in a low resource setting. Investigations like Ultrasound, Computed Tomography and Magnetic Resonance Imaging are costly. Hence, we require a simple easy method to assess obesity.

Researcher Jean Vague was the first to put substantiate the relation between fat distribution and different type of body Mass Index and its association with health risks. He associated Neck Circumference in men for upper body fat distribution. Sjostrom et al stated that NC is an index for subcutaneous adipose tissue distribution of the upper body and its relation to cardiovascular risk factors. $\mathrm{He}$ also stated that free fatty acid release from upper body subcutaneous fat was larger than from the lower body subcutaneous fat. The inference from this is that $\mathrm{NC}$ can be used as an index to predict Metabolic Syndrome.

This study confirmed the existence of a strong correlation between NC and BMI, Waist Circumference, Hip Circumference and Waist-to-Hip Ratio. Hence, $\mathrm{NC}$ can be used to identify overweight and obesity. The results of the present study correlate well with a similar study where it was stated that there were significant and positive correlations between BMI-WC, BMI-NC and WC and NC $\left(\mathrm{p}<0.001\right.$ for overweight and obesity ${ }^{7}$. $\mathrm{NC}$ can be used as a simple and timesaving screening measure to identify overweight and obese patients. Neck Circumference showed significant correlation with waist circumference- $r=0.64$, BMI- $r=0.66$ ) and body fat $\%$ $0.45^{8}$. In this study, mean $\mathrm{NC}$ of normal weight was 34.02 $\mathrm{cm}$. In another study ${ }^{9}$ the mean $\mathrm{NC}$ of the subjects with normal weights was $33.5 \pm 3.4 \mathrm{~cm}$ for the women and identified $\geq 34.75 \mathrm{~cm} \mathrm{NC}$ as the best cut off for identifying subjects with central obesity. In this study, these cut-off levels for NC were associated with a significantly increased risk for diabetes, dyslipaedemia, and hypertension. In this study, mean NC was $36.4 \mathrm{~cm}$ associated with Metabolic Syndrome.

In the investigations carried out another study ${ }^{10} \mathrm{NC}$ was associated with most of the cardiovascular disease risk factors like WC, HC, WHR. They also correlated with SBP, HDL and insulin-related indices, which is similar to the conclusions drawn from this study where NC has positive correlation with all parameters of Metabolic Syndrome, namely systolic blood pressure, diastolic blood pressure, fasting blood sugar and total cholesterol, HDL, LDL and Triglycerides.

NC improves the prediction of incident cardiovascular disease risk factors over BMI and waist circumference ${ }^{11}$. This conjecture is also established in this study, since NC shows strong correlation with all parameters of Metabolic Syndrome. As such, adding NC to other anthropometry measurements will appreciably increase the prediction of Metabolic Syndrome. 
It was proposed that fat in the upper body deposition in pre-menopause women is due to unbound androgens which causes enlargement of abdominal adipocytes resulting in altered glucoseinsulin homeostasis ${ }^{12}$. In this study it was found that abnormal NC and metabolic syndrome are seen more in peri-menopause age group (24\%) compared to those of the post menopause age group.

\section{Conclusion}

Neck Circumference can be used as a simple and easy screening test to assess obesity. When NC increases, Metabolic Syndrome can be predicted. Menopause transition may predispose women to Metabolic Syndrome. Hence, simple screening test like NC is needed to predict Metabolic Syndrome. Our study point to the fact that as $\mathrm{NC}$ increases its association is positive with metabolic syndrome in midlife transition. Hence, preventive measure can be taken during this period.

\section{Acknowledgement}

The author thanks the Director, Prof. Saveetha Rajesh for permitting to conduct this study and also thanks the Dean Prof. Gunasagaran for the encouragement and support.

\section{References}

1. Svendsen OL, Hassager C, Christiansen C. Age-and menopause-associated variations in body composition and fat distribution in healthy women as measured by dual-energy X-ray absorptiometry. Metabolism. 1995; 44(3):369-73. https://doi.org/10.1016/00260495(95)90168-X

2. Poehlman ET. Menopause, energy expenditure and body composition. Acta Obstetrics Gynaecologica Scandinavica. 2002; 81(7):603-11. PMid: 12190834. https://doi.org/10.1080/ j.1600-0412.2002.810705.x

3. Lim S, Meigs JB. Ectopic fat and cardiometabolic and vascular risk. International Journal of Cardiology. 2013; 19(3):166-7. PMid: 24063931. https://doi.org/10.1016/j. ijcard.2013.08.077
4. Nielsen S, Guo Z, Johnson CM, Hensrud D, Jensen MD. Splanchnic lipolysis in human obesity. Journal of Clinical Investigations. 2014; 113(11):1582. PMid: 15173884 PMCid: PMC419492. https://doi.org/10.1172/JCI21047

5. Ectopic fat depots and cardiovascular disease circulation. 2011. https://www.ahajournals.org/doi/10.1161/ CIRCULATIONAHA.111.077602

6. Stabe C, Vasques AC, Lima MM, Tambascia MA, Pareja JC, Yamanaka A. Neck Circumference as a simple tool for identifying the metabolic syndrome and insulin resistance: Results from the Brazilian Metabolic Syndrome Study. Clinical Endocrinology (OXF). 2013; 78:874-81. PMid: 22804918. https://doi.org/10.1111/j.1365-2265.2012.04487.x

7. Hatipoglu N, Mazicioglu MM, Kurtoglu S, Kendirci M. Neck Circumference: An additional tool of screening overweight and obesity in childhood. European Journal of Pediatrics. 2010; 169(6):733-9. PMid: 19936785. https:// doi.org/10.1007/s00431-009-1104-Z

8. Neck Circumference may be a better alternative to standard anthropometric measures. 2016. https://www.ncbi.nlm.nih. gov/pubmed/26981543

9. Alfadhli EM, Sandokji AA, Zahid BN, Makkawi MA, Alshenaifi RF, Thani TS, Habeeb HA. Neck Circumference as a marker of obesity and a predictor of cardiometabolic risk among Saudi subjects. Saudi Medical Journal. 2017; 38(12):1219-23. PMid: 29209671 PMCid: PMC5787633. https://doi.org/10.15537/smj.2017.12.20926

10. Androutsos O, Grammatikaki E, Moschonis G, RomaGiannikou E, Chrousos GP, Manios Y, Kanaka-Gantenbein C. Neck Circumference: A useful screening tool of cardiovascular risk in children. Pediatric Obesity. 2012; 7(3):187-95. PMid: 22505226. https://doi.org/10.1111/ j.2047-6310.2012.00052.x

11. Preis SR, Pencina MJ, D’Agostino RB Sr, Meigs JB, Vasan RS, Fox CS. Neck Circumference and the development of cardiovascular disease risk factors in the Framingham Heart Study. Diabetes Care. 2013; 36(1):e3-e3. PMid: 23264305 PMCid: PMC3526209. https://doi.org/10.2337/ dc12-0738

12. Evans DJ, Hoffmann RG, Kalkhoff RK, Kissebah AH. Relationship of androgenic activity to body fat topography, fat cell morphology and metabolic aberrations in premenopausal women. The Journal of Clinical Endocrinology and Metabolism. 1983; 57(2):304-10. PMid: 6345569. https://doi.org/10.1210/jcem-57-2-304 